\title{
Fabrication of Fork-Shaped Retinal Stimulator Integrated with CMOS Microchips for Extension of Viewing Angle
}

\author{
Toshihiko Noda*, Kiyotaka Sasagawa, Takashi Tokuda, \\ Hiroyuki Kanda ${ }^{1}$, Yasuo Terasawa ${ }^{2}$, Hiroyuki Tashiro ${ }^{3}$, \\ Takashi Fujikado ${ }^{1}$ and Jun Ohta \\ Nara Institute of Science and Technology, 8916-5 Takayama, Ikoma, Nara 630-0192, Japan \\ ${ }^{1}$ Osaka University, 2-2 Yamadaoka, Suita, Osaka 565-0871, Japan \\ ${ }^{2}$ NIDEK Co., Ltd., 13-2 Hama-cho, Gamagori, Aichi 443-0038, Japan \\ ${ }^{3}$ Kyushu University, 3-1-1 Maidashi, Higashi-ku, Fukuoka 812-8582, Japan
}

(Received February 27, 2014; accepted July 30, 2014)

Key words: retinal stimulation, viewing angle, fork-shaped stimulator, CMOS microchip, in vivo experiment

A fork-shaped retinal stimulator for a wide viewing angle was fabricated and demonstrated. This stimulator has two flexible fork elements, each with a stimulus head. The two fork elements can be implanted at different places in the eyeball to cover a wider area of the retina. The stimulus head has stimulus electrodes with the same shape as the electrodes utilized in a clinical trial for six weeks. Complementary metal-oxide semiconductor (CMOS) microchips were integrated into the stimulator and mounted next to the stimulus electrodes. Since stimulus current generators are integrated into the microchips, no external wires for stimulus current are required. A multiplexer, which reduces the number of wirings, was also integrated into the microchips. The fabricated stimulator was evaluated through in vivo animal experiments. The stimulator was successfully implanted in two places of the eyeball. Retinal stimulation by the device evoked nerve responses from optic chiasma and produced a specific peak of evoked potential. Experimental results demonstrate the proof of concept of a fork-shaped stimulator and suggest that the same device configuration can realize multiple forkshaped stimulators for a wider viewing angle. Moreover, increasing the number of fork elements of the multifork stimulator without additional wirings becomes possible.

*Corresponding author: e-mail: t-noda@ms.naist.jp 


\section{Introduction}

A retinal prosthesis is a candidate device for blind patients to regain their vision. Particularly in the cases of retinitis pigmentosa (RP) and age-related macular degeneration (AMD), although photoreceptor cells are degenerated, some of the retinal cells that include ganglion cells remain. Retinal stimulation can be applicable to these diseases. Electrical stimulation of the remaining cells evokes visual sensation as phosphene.

There are three methods of retinal stimulation as shown in Fig. 1. Epi-retinal stimulation $^{(1,2)}$ has a lower threshold of electrical stimulation. A retinal stimulator faces the retina from the inner side of the eyeball. This configuration causes difficulty in fixing the retinal stimulator. In sub-retinal stimulation, ${ }^{(3-6)}$ the fixation of the retinal stimulator is tighter than that in epi-retinal stimulation. However, surgical operation, which is the insertion of the retinal stimulator under the retina, is difficult. In suprachoroidaltransretinal stimulation (STS) ${ }^{(7,8)}$ which is the third method, the position of the retinal stimulator is very different from those in the other methods. The retinal stimulator, which is implanted outside of the choroid, does not touch the retina directly. Therefore, intraocular surgery is not required. Invasiveness is lower and safety is higher than the other methods that require intraocular surgery. In the retinal prosthesis, viewing angle is also as important as resolution. As explained in the next section, in the case of the STS method, large-area stimulation of the retina is possible by the implantation of a large retinal stimulator that covers a large area of the eyeball.

Our research group has been focusing on the STS method. A prototype of the retinal prosthetic system used in the STS method was developed successfully, and a semiacute clinical trial was performed..$^{(9,10)}$ As a next-generation retinal stimulator, a complementary metal-oxide semiconductor (CMOS) integrated retinal stimulator was also proposed and demonstrated.(11-15) The possibility of realizing super-multielectrode arrays, such as an array of one thousand electrodes, was proved using CMOS technology.

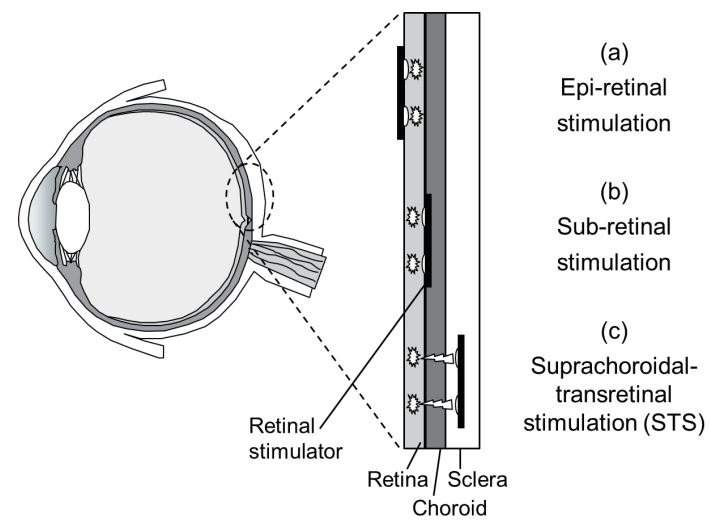

Fig. 1. Three approaches for retinal stimulation. (a) Epi-retinal stimulation. (b) Sub-retinal stimulation. (c) STS. 
In the STS method, the distance between the retina and the stimulus electrodes is larger than those in the other methods. This distance results in a higher threshold of the stimulation as a result of the spreading of the stimulus current. Typical threshold current ranges from several tens of $\mu \mathrm{A}$ to a few hundreds of $\mu \mathrm{A}$. Large electrodes are necessary to allow higher stimulus currents. In a semiacute clinical trial, bullet-shaped electrodes of $500 \mu \mathrm{m}$ height and diameter were adopted. Also, in the case of CMOS-based retinal stimulators, an array of larger electrodes was fabricated using stimulus electrodes, which were the same as the electrodes utilized in the clinical trial.(9,10) The electrodes were hybrid-mounted to a flexible substrate with CMOS microchips. Bulk electrodes become smart electrodes through the integration of CMOS microchips, and they obtain functions of a multiplexer and a stimulus current generator. The smart-wiring technology, which is the integration of individual microchips into the electrode, realizes a reduction in the number of wires used. The reduction in the number of wires is a critical problem in realizing a multichannel stimulator.

For expansion of the viewing angle, the integration of CMOS microchips is suitable because a larger retinal stimulator that covers a wider area of the retina has a larger number of electrodes. However, even if a larger stimulator can be developed, implantation of the large stimulator to the eyeball becomes complicated because of interference with blood vessels and nerve bundles. To solve this problem, we propose an approach that covers the eyeball with a multifork stimulator as shown in Fig. 2. Stimulus electrodes are mounted on a flexible multifork-shaped substrate. Each fork element can be implanted easily and gaps between the forks can be utilized to avoid interference with blood vessels and nerve bundles. As a first step to develop a multifork stimulator, a prototype of a fork-shaped stimulator was fabricated in this study. As shown in Fig. 3 , a simple forked stimulator with two stimulus heads was fabricated to demonstrate the proposed concept. The integration of CMOS microchips was performed, and dedicated microchips were designed and fabricated. The fabrication process was optimized, and then the stimulator was fabricated using the optimized process. The fabricated stimulator was evaluated through animal experiments for functional validation.
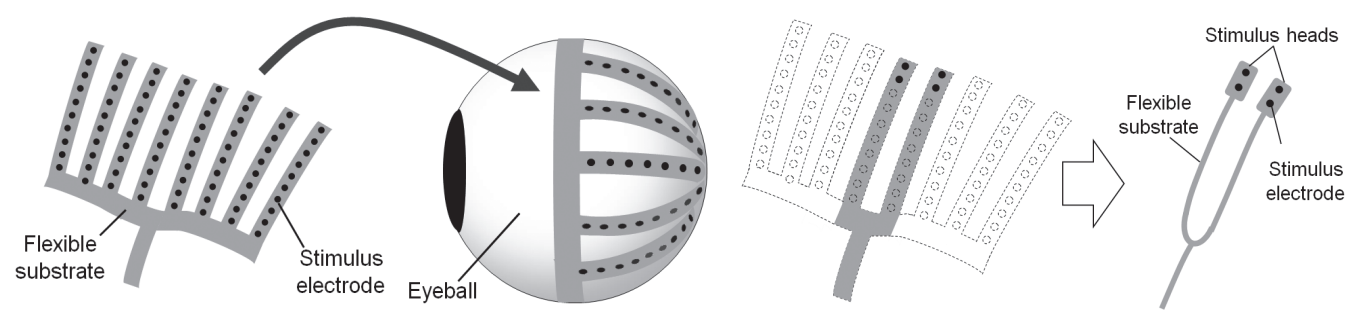

Fig. 2 (left). Conceptual figure of multifork stimulator.

Fig. 3 (right). Prototype of fork-shaped stimulator. 


\section{Device Structure}

\subsection{Overview of fork-shaped retinal stimulator}

Figure 4 shows an overview of the fork-shaped retinal stimulator. A flexible printed circuit (FPC) made from a polyimide film is used as the flexible substrate of the stimulator. Other elements of the stimulator, which are stimulus electrodes and CMOS microchips, are mounted on the flexible substrate. The stimulator is $20 \mathrm{~cm}$ long and is divided into two fork elements at the center. The tips of the elements are stimulus heads with stimulus electrodes. In this study, only two electrodes are mounted on the stimulus head to perform animal experiments as functional validation. A small head is required for experimental animals with smaller eyeballs than humans. If a larger stimulus head is allowable, more electrodes should be mounted on the stimulus head using the same device configuration.

The cross-sectional structure of the stimulus head is shown in Fig. 5. The fundamental structure is the same as in our previous works ${ }^{(16,17)}$ however, a different method for the fixation of the stimulus electrode is used. Stimulus electrodes and CMOS microchips are connected to each other. The stimulus electrodes are controlled by CMOS microchips that supply stimulus current.

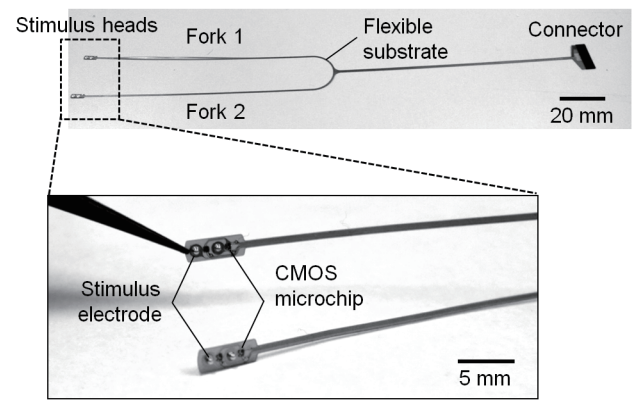

Fig. 4. Overview of fork-shaped stimulator.

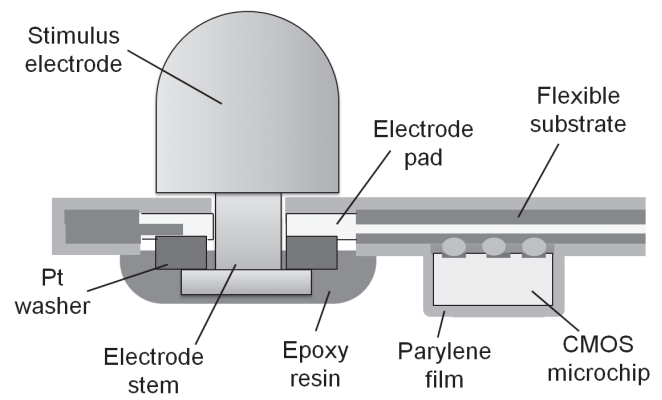

Fig. 5. Cross-sectional structure of stimulus head. 


\subsection{CMOS microchip}

Dedicated CMOS microchips serve as controllers of the stimulus electrode and stimulus current generator. The basic architecture of the microchips used in this work is the same as that in the previous work. ${ }^{(15)}$ As shown in Fig. 6, all of the microchips mounted on the retinal stimulator are connected to the common bus line for control signal and power supply. The number of bus lines is not changed by the number of microchips. In this work, four microchips were connected to the common bus line. The microchips were designed and fabricated with $0.35 \mu \mathrm{m}$ standard CMOS technology. The die size is $400 \mu \mathrm{m}$ sq. as shown in Fig. 7.

In the previous work, ${ }^{(15)}$ an intrinsic chip ID number, which is used for the selection of a control target, was programmed in the chip design step. Therefore, it was not practical to provide many different ID numbers, because many different chip designs should be prepared. To solve this problem, we propose chip ID writing after CMOS chip fabrication. The chip ID number can be programmed by cutting fuse lines by laser processing as shown in Fig. 7.

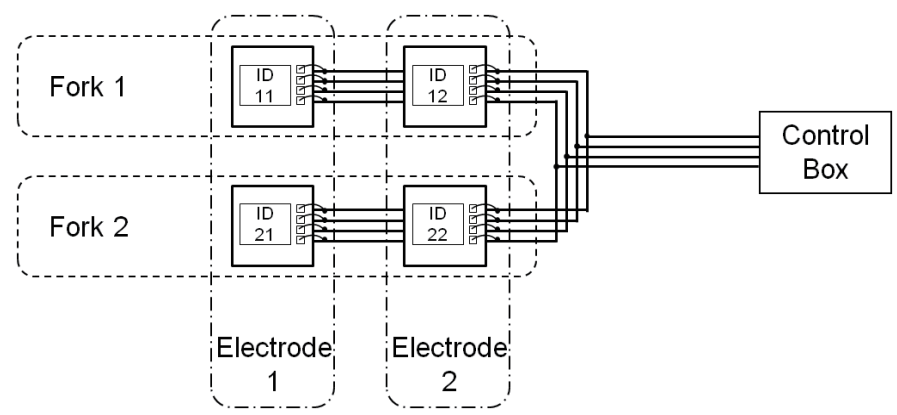

Fig. 6. Connection of microchips through common bus line.

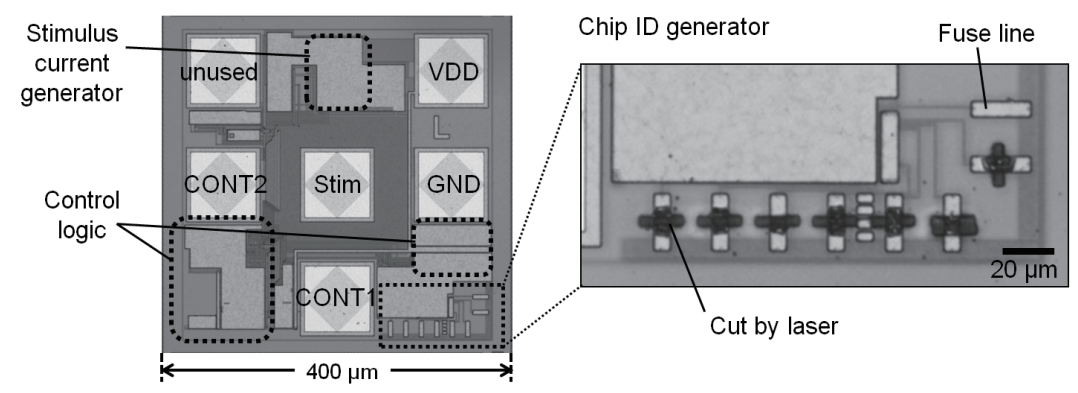

Fig. 7. Photograph of CMOS microchip. The enlarged micrograph shows a chip ID generator. 


\subsection{Stimulus electrode}

Figure 8 shows the stimulus electrode. The electrode is made of platinum and has the same bullet shape as the electrodes utilized in a semiacute clinical trial.(9,10) The electrodes of $500 \mu \mathrm{m}$ height and diameter are fabricated by a machining process using a precision lathe. At the bottom of the electrodes, a stem is formed to fix the electrodes to a flexible substrate. Platinum washers (Fig. 9) made from a $20-\mu \mathrm{m}$-thick platinum film are also used to fix the electrodes. The fabrication process is explained in the following chapter in detail.

\section{Device Fabrication}

The fork-shaped retinal stimulator was fabricated through the process steps shown in Fig. 10. Details of the fabrication process are shown as follows.

1. Chip ID writing

Fuse lines, which define the chip ID number, were cut by laser processing. 8-Bit binary ID numbers were assigned to each microchip to be mounted on the stimulator.

2. Stud bump formation on CMOS die

Au stud bumps for flip-chip bonding were formed on the CMOS die. The diameter of the stud bumps is $80 \mu \mathrm{m}$.

3. Chip separation

The CMOS die was scribed to the microchips by a mechanical dicing process.

4. Leveling of electrode pads of flexible substrate

$\mathrm{Au}$ stud bumps were formed on electrode pads of the flexible substrate to be used for connecting the CMOS microchips. Then, the bumps formed were tamped and leveled by pressing a flat glass.

5. Flip-chip bonding of CMOS microchips

The CMOS microchips were mounted on the flexible substrate by flip-chip bonding technology. Anisotropic conductive paste (TAP0401C, KYOCERA Chemical Corporation) was used for sure connection and mechanical reinforcement.
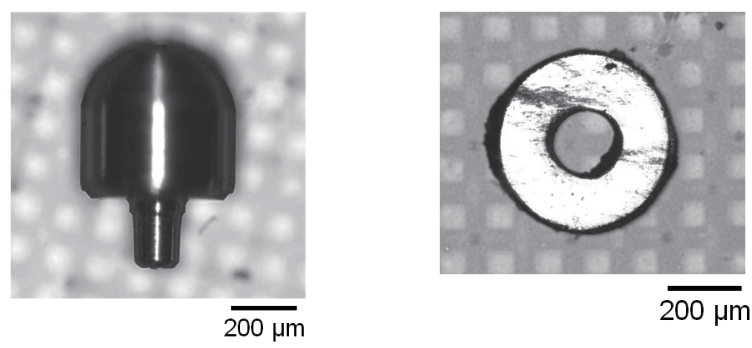

Fig. 8 (left). Bullet-shaped stimulus electrode. Fig. 9 (right). Pt washer for electrode fixation. 

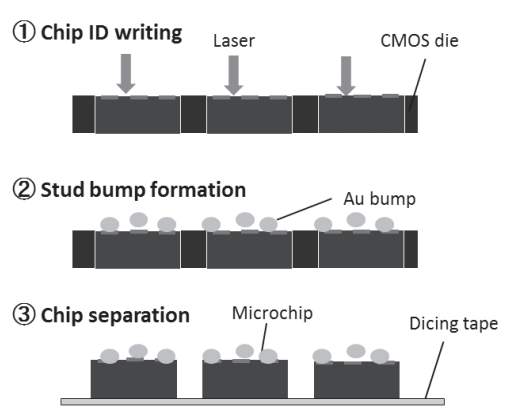

(4) Leveling of electrode pads

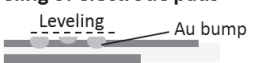

(5) Flip-chip bonding

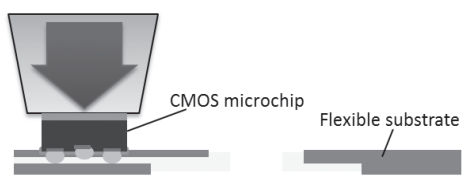

6) Waterproof coating Parylene film

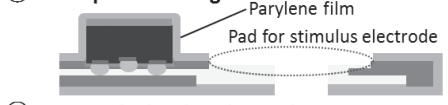

(7) Mount of stimulus electrode

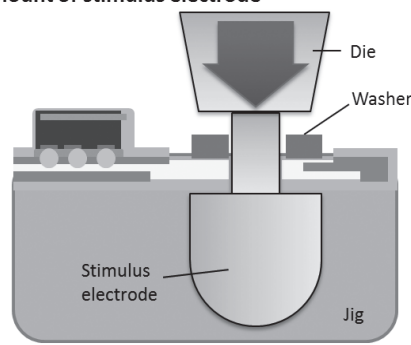

(8) Insulation of stimulus electrode Epoxy resin

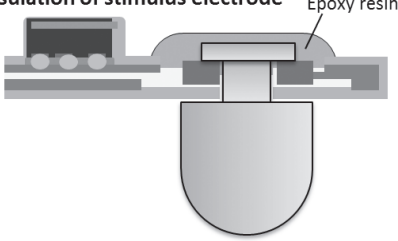

Fig. 10. Fabrication process for stimulator.

6. Waterproof coating

The flexible substrate where the CMOS microchips were mounted was entirely coated by parylene-C for waterproofing and biocompatibility. ${ }^{(18)}$

7. Mounting of stimulus electrodes

After the coating, the electrode pads for stimulus electrodes were covered with a parylene film. To remove the parylene film from the electrode pads, laser processing was performed. Each stimulus electrode was set to a jig, and then the stem of the stimulus electrode was passed through a hole of the flexible substrate. After setting a Pt washer to the stem, the stem was crimped by a die. The stimulus electrode was fixed mechanically and connected electrically to the flexible substrate.

8. Insulation of stimulus electrodes

The crimped side of the electrodes was covered with epoxy resin for waterproofing.

The fabricated retinal stimulator is shown in Fig. 11. Figure 11(a) shows the front side of the stimulus head. Four electrodes were mounted on the flexible substrate. Some white patterns such as scratches are halations of the flexible substrate surface. Figure 11(b) shows the back side of the stimulus head. CMOS microchips were mounted next to the stimulus electrodes. In an enlarged photograph, the crimped stem of the electrode can be confirmed. The perimeter of CMOS microchips was surrounded by anisotropic conductive paste. Figure 11(c) shows the side view of the stimulus head. The bullet shape of the electrode can be observed in the photograph. The interval of the two electrodes mounted on the stimulus head is $2 \mathrm{~mm}$. 


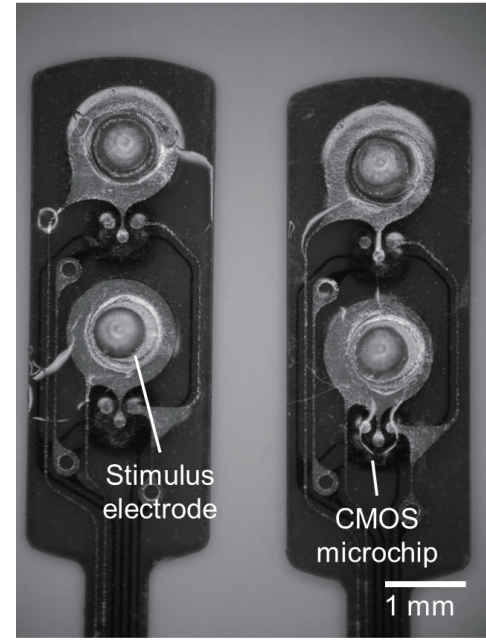

(a) Front view
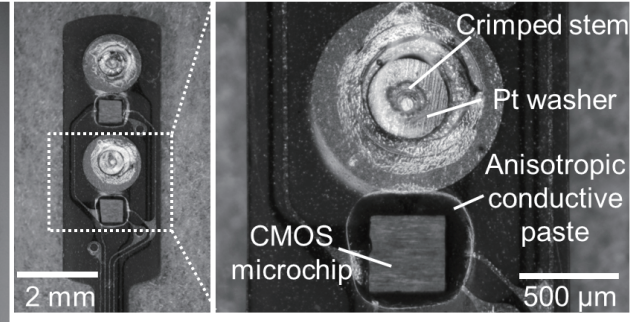

(b) Back view

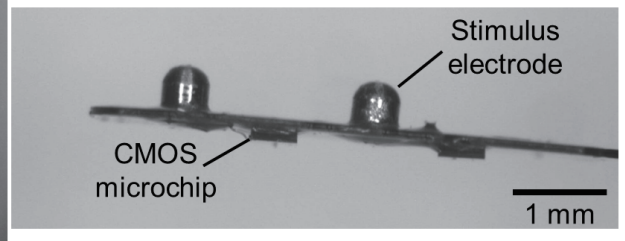

(c) Side view

Fig. 11. Photographs of stimulus heads of fabricated stimulator. (a) Front view. (b) Back view. (c) Side view.

\section{Functional Validation}

\subsection{Experimental setup}

Functional validation of the fabricated stimulator was performed through in vivo animal experiments. All of the animal experiments were regulated by the guidelines of Osaka University for animal experiments. Figure 12 shows the configuration of the in vivo animal experiment. An anesthetized cat was used for this animal experiment. Two stimulus heads were implanted to the eyeball. The connector of the fabricated stimulator was connected to a control box. The control box, which supplies DC power and provides control signals to the CMOS microchips mounted on the stimulator, was linked to a laptop computer. A bipolar electrode for recording was inserted into the optic chiasma and connected to a recorder through a differential amplifier. ${ }^{(19)}$ The timing pulse of stimulation was generated by the control box and was sent to the recorder as a trigger signal to start recording.

\subsection{Surgery}

Two stimulus heads were implanted to the eyeball. Intrascleral pockets for implantation were formed at two different positions. Each stimulus head was inserted into each intrascleral pocket as shown in Fig. 13. After the surgery, ophthalmoscopy was performed. Figure 14 shows a photograph of the fundus. The positions of the stimulus electrodes can be confirmed clearly from this photograph. 


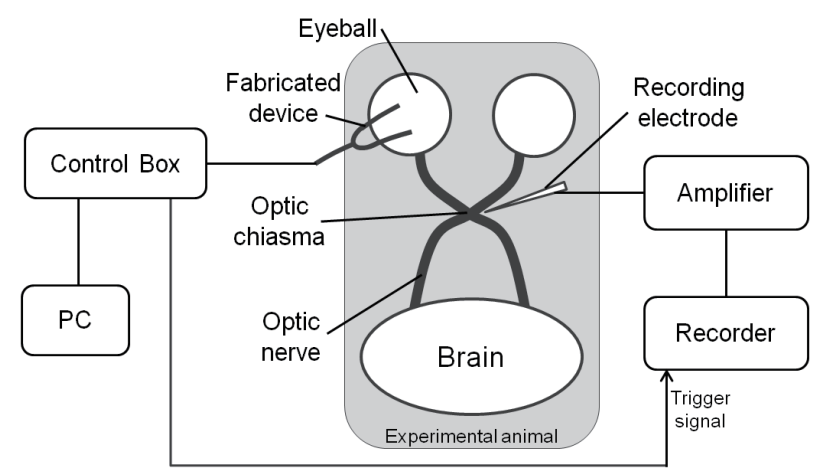

Fig. 12. Experimental setup of in vivo experiments.
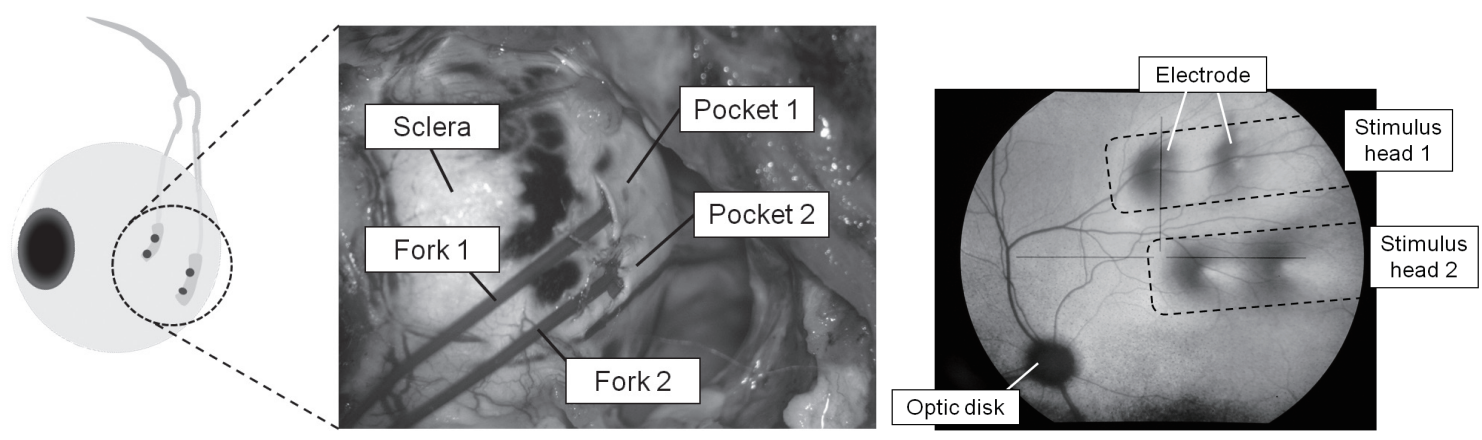

Fig. 13 (left). Implantation of stimulator to eyeball.

Fig. 14 (right). Fundus photograph after implantation.

\subsection{Electrophysiological experiment}

The neural response evoked by the retinal stimulation was measured. A cathodic first biphasic pulse was used for the stimulation. The duration of both the cathodic and anodic pulses was $500 \mu$ s with no interpulse duration. The stimulus current was varied to 75,140 , and $330 \mu \mathrm{A}$. The neural response evoked by the retinal stimulation was observed at the optic chiasma. The output of the amplifier connected to a recording electrode was recorded by a recorder.

Figure 15 shows the results of the electrophysiological measurement. In the cases of 140 and $330 \mu \mathrm{A}$ stimulations, a specific waveform after the stimulation was observed. The latency of the specific peak observed after the stimulation is the same in both cases. On the other hand, no specific peak was observed in the case of $75 \mu$ A stimulation. From the results, the threshold of the retinal stimulation was determined to be higher than 75 $\mu \mathrm{A}$ and lower than $140 \mu \mathrm{A}$. By comparing the results of 140 and $330 \mu \mathrm{A}$ stimulations, a strong stimulation is considered to evoke a large response. Thus, in our in vivo animal experiments, the fabricated stimulator was successfully implanted to the eyeball and the stimulus function was also demonstrated successfully. 


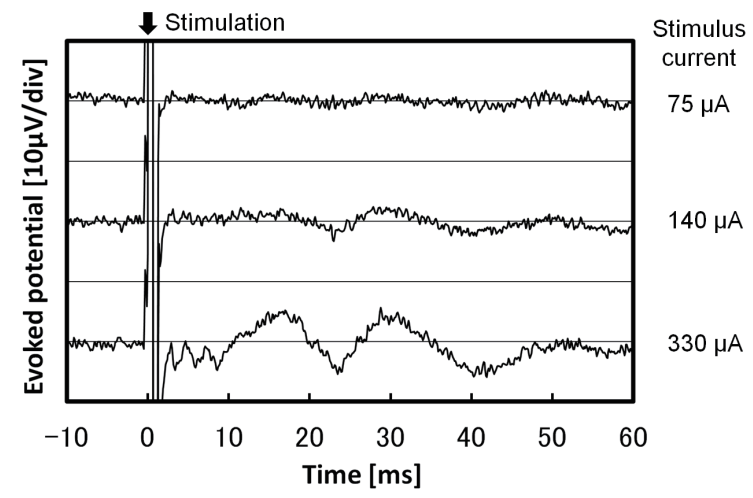

Fig. 15. Results of electrophysiological measurement.

\section{Conclusions}

A retinal stimulator for a retinal prosthesis that realizes expansion of the viewing angle was described in this paper. As one of the approaches, a multifork retinal stimulator was proposed. A prototype of a fork-shaped retinal stimulator with two fork elements was fabricated to demonstrate the proposed concept. Smart electrodes integrated with CMOS microchips were utilized in the stimulator. The integration of CMOS microchips controls the stimulator with few wires even if the number of stimulus electrodes is increased.

A dedicated CMOS microchip was designed and fabricated. This microchip functions as a multiplexer and a stimulus current generator. The chip ID number can be written arbitrarily after the chip fabrication by laser processing. CMOS microchips were mounted onto a flexible substrate using flip-chip bonding technology. Stimulus electrodes with the same bullet shape as the electrodes used in a semiacute clinical trial were mounted next to the CMOS microchips. An in vivo animal experiment was performed as a functional validation of the fabricated stimulator. The stimulator was implanted to the eyeball, and current-controlled retinal stimulation was performed. The responses to the stimulation were observed at the optic chiasma. The evoked potential of nerve responses was observed clearly as a result of retinal stimulation.

The proposed concept of the fork-shaped stimulator was successfully demonstrated. The fabricated simple fork stimulator has expandability toward multifork stimulators with the same configuration. To realize multifork stimulators, the evoked area of the retina stimulated by one electrode should be investigated. The spread of stimulation can be discussed through an estimation of the evoked retina by optical imaging of the fundus. ${ }^{(19,20)}$ Miniaturization of stimulus electrodes should be realized for multifork stimulators to mount a large number of electrodes. Miniaturization of the electrodes can be achieved by surface coating of the electrodes. ${ }^{(21,22)}$ Owing to these technologies, a wide-view retinal prosthesis might be achieved using multifork stimulators. 


\section{Acknowledgements}

Part of this study stems from the "Brain Machine Interface Development" study carried out under the Strategic Research Program for Brain Sciences by the Ministry of Education, Culture, Sports, Science and Technology of Japan. It is also supported by a Grant-in-Aid for Young Scientists (B), \#23760312, from the Japan Society for the Promotion of Science.

\section{References}

1 M. S. Humayun, J. D. Weiland, G. Y. Fujii, R. Greenberg, R. Williamson, J. Little, B. Mech, V. Cimmarusti, G. Van Boemel, G. Dagnelie and E. De Juan,: Vision Research 43, (2003) 2573.

2 L. da Cruz, B. F. Coley, J. Dorn, F. Merlini, E. Filley, P. Christopher, F. K. Chen, V. Wuyyuru, J. Sahel, P. Stanga, M. Humayun, R. J. Greenberg, and G. Dagnelie: Br. J. Ophthalmol. 97 (2013) 632.

3 E. Zrenner: Science 295 (2002) 1022.

4 E. Zrenner, K. U. Bartz-Schmidt, H. Benav, D. Besch, A. Bruckmann, V. P. Gabel, F. Gekeler, U. Greppmaier, A. Harscher, S. Kibbel, J. Koch, A. Kusnyerik, T. Peters, K. Stingl, H. Sachs, A. Stett, P. Szurman, B. Wilhelm and R. Wilke: Proc. R. Soc. B Biol. Sci. 278 (2010) 1489.

5 D. B. Shire, S. K. Kelly, J. C. J. Chen, P. Doyle, M. D. Gingerich, S. F. Cogan, W. A. Drohan, O. Mendoza, L. Theogarajan, J. L. Wyatt and J. F. Rizzo: Development and Implantation of a Minimally Invasive Wireless Subretinal Neurostimulator 56 (2009) 2502.

6 K. Mathieson, J. Loudin, G. Goetz, P. Huie, L. Wang, T. I. Kamins, L. Galambos, R. Smith, J. S. Harris, A. Sher and D. Palanker: Nat. Photonics 6 (2012) 391.

7 H. Kanda, T. Morimoto, T. Fujikado, Y. Tano, Y. Fukuda and H. Sawai: Invest. Ophthalmol. Vis. Sci. 45 (2004) 560.

8 T. Fujikado, T. Morimoto, H. Kanda, S. Kusaka, K. Nakauchi, M. Ozawa, K. Matsushita, H. Sakaguchi, Y. Ikuno, M. Kamei and Y. Tano: Graefes Arch. Clin. Exp. Ophthalmol. Albr. von Graefes Arch. fur Klin. und Exp. Ophthalmol. 245 (2007) 1411.

9 T. Fujikado, M. Kamei, H. Sakaguchi, H. Kanda, T. Morimoto, Y. Ikuno, K. Nishida, H. Kishima, T. Maruo, K. Konoma, M. Ozawa and K. Nishida: Investigative Ophthalmology \& Visual Science 52 (2011) 4726.

10 T. Fujikado, M. Kamei, H. Sakaguchi, H. Kanda, T. Morimoto, Y. Ikuno, K. Nishida, H. Kishima, T. Maruo, H. Sawai, T. Miyoshi, K. Osawa and M. Ozawa: Sensors Mater. 24 (2012) 181.

11 J. Ohta, N. Yoshida, K. Kagawa and M. Nunoshita: Jpn. J. Appl. Phys. 41 (2002) 2322.

12 T. Tokuda, R. Asano, S. Sugitani, M. Taniyama, Y. Terasawa, M. Nunoshita, K. Nakauchi, T. Fujikado, Y. Tano and J. Ohta: Jpn. J. Appl. Phys. 47 (2008) 3220.

13 T. Tokuda, K. Hiyama, S. Sawamura, K. Sasagawa, Y. Terasawa, K. Nishida, Y. Kitaguchi, T. Fujikado, Y. Tano and J. Ohta: CMOS-Based Multichip Networked Flexible Retinal Stimulator Designed for Image-Based Retinal Prosthesis 56 (2009) 2577.

14 T. Tokuda, Y. Takeuchi, Y. Sagawa, T. Noda, K. Sasagawa, K. Nishida, T. Fujikado and J. Ohta: Development and in vivo Demonstration of CMOS-Based Multichip Retinal Stimulator With Simultaneous Multisite Stimulation Capability 4 (2010) 445.

15 T. Noda, K. Sasagawa, T. Tokuda, Y. Terasawa, H. Tashiro, H. Kanda, T. Fujikado, and J. Ohta: Electron. Lett. 48 (2012) 1328.

16 T. Noda, K. Sasagawa, T. Tokuda and J. Ohta: "Flexible Retinal Prosthesis Device with CMOS Micorochip," in CMOS-Emerging Technology 2012, 2012. 
17 T. Noda, K. Sasagawa, T. Tokuda and J. Ohta: "A Smart Electrode Array Devices with CMOS Microchip for Neural Interface," in International Conference on BioElectornics, BioSensors, Biomedical Devices, BioMEMS/NEMS and Applications 2012 (Bio4Apps 2012), 2012, p. Oral Presentation 8.

18 B. D. Winslow, M. B. Christensen, W.-K. Yang, F. Solzbacher and P. A. Tresco: Biomaterials 31 (2010) 9163.

19 Y. Okawa, T. Fujikado, T. Miyoshi, H. Sawai, S. Kusaka, T. Mihashi, Y. Hirohara and Y. Tano: Invest. Ophthalmol. Vis. Sci., vol. 48 (2007) 4777.

20 Y. Hirohara, T. Mihashi, H. Kanda, T. Morimoto, T. Miyoshi, J. S. Wolffsohn and T. Fujikado: Experimental eye research 109 (2013) 1.

21 Y. Pan, T. Noda, K. Sasagawa, T. Tokuda, H. Kanda, T. Fujikado and J. Ohta: J. Phys. Conf. Ser. 352 (2012) 012005.

22 Y.-L. Pan, T. Noda, K. Sasagawa, T. Tokuda and J. Ohta: IEEJ Trans. Electr. Electron. Eng. 8 (2013) 310 . 